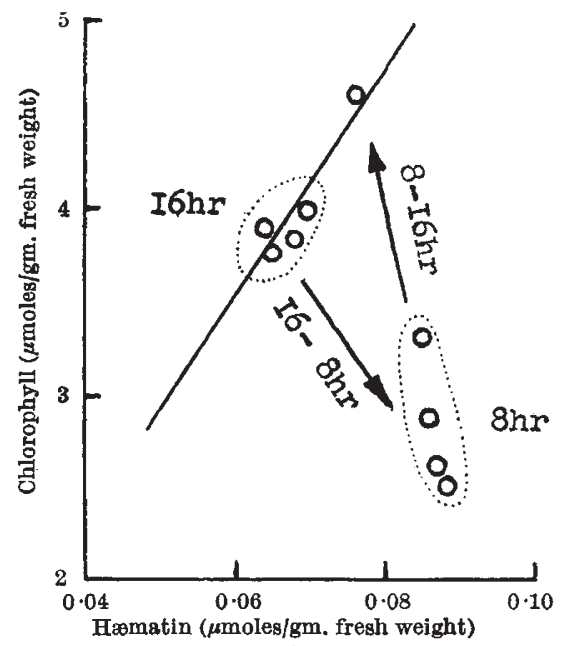

Fig. 2. Effect of transfer from long to short days and vice versa on chlorophyll/hmmatin ratio in hemp. (The arrows indicate

Further details of these experiments will be published elsewhere. I am greatly indebted to Dr. R. Hill for valuable help in the method of measuring hæmatin in leaves, to Prof. F. G. Young for providing facilities in the Department of Biochemistry, Cambridge, to Mr. J. Gilmour, director of the Botanical Garden, Cambridge, for growing the plants, and to the I.R.S.I.A. (Belgium) for financial support.

Department of Biochemistry, C. SironvaI Cambridge, and

Laboratory of Plant Physiology,

Centre des Hormones Végétales, Liège. Aug. 1.

${ }^{1}$ Sironval, C., C.R. Rech. IRSIA, Bruxelles, 18, 1 (1957).

${ }^{2}$ Cheuvart, C., Bull. Acad. Roy. Belg., 40, 1152 (1954).

s Cajlahjan, M., and Bavrina, T., Phys. Rast., 4, 312 (1957).

- Sironval, C., C.R. $2^{\bullet}$ Congrès de Photobiologie, Torino, 387.

s Sironval, C., Bonnier, C., and Verlinden, J.-P., Phys. Plant, 10, 697 (1957).

- Sironval, C., Nature, 181, 1272 (1958).

' Hill, R., and Scarisbrick, R., New Phytol., 50, 98 (1951).

Mackinney, G., J. Biol. Chem., 140, 315 (1941).

\section{Occurrence of Coumarin Analogues in Lemon Juice}

Threre has been little or no investigation of coumarin compounds in lemon juice although their presence in lemon oil has been well established ${ }^{1-4}$.

It was of interest to determine whether coumarin analogues were indeed present in lemon juice. Petroleum ether extracts of California lemon juice concentrates were examined by the chromatostrip technique of Miller and Kirchner ${ }^{5}$. This revealed a group of eight fluorescent spots suggestive of the coumarin array demonstrated by Stanley and Vannier, ${ }^{4,6}$. Extracts were then subjected to downward chromatostrip development ${ }^{6}$ and separation of four of the compounds was achieved in amounts adequate for qualitative ultra-violet spectral measurements. Comparison of $R_{F}$ values with known coumarin compounds and ultra-violet absorption spectra tentatively identified these four compounds as 5-geranoxypsoralen (bergamottin), 5-geranoxy-7methoxycoumarin, 8-geranoxypsoralen and 5,7-dimethoxycoumarin (limettin) (Table 1).

The possibility remained that the coumarin compounds detected in the juice could conceivably be
Table 1

\begin{tabular}{|l|c|c|}
\hline \multicolumn{1}{|c|}{ Compound } & $R_{F} \times 100^{*}$ & $\begin{array}{c}\text { Ultra-violet absorp- } \\
\text { tion maxima } \\
(\mathrm{m} \mu)\end{array}$ \\
\hline 5-Geranoxypsoralen & 68 & $251,259,269,310$ \\
5-Geranoxy-7-methoxycoumarin & 64 & $246,256,325$ \\
8-Geranoxypsoralen & 40 & $244,250,265,300$ \\
5,7-Dimethoxycoumarin & 25 & $246,256,325$ \\
Oxypeucedanin hydrate & 5 & $250,259,268,310$ \\
Byakangelicin & 0 & $\mathbf{2 4 1 , 2 4 9 , 2 7 0 , 3 1 3}$ \\
\hline On silicic acid chromatostrips (refs. 5, 6). Irrigation solvent:
\end{tabular}

25 per cent ethyl acetate in $n$-hexane (v/v).

present because of inclusion of lemon oil in the concentrate. This could come about during the mechanical reaming of the fruit resulting from the rupture of the oil sacs releasing small amounts of oil into the juice ${ }^{7}$.

In order to determine whether these coumarin compounds were present in oil-free lemon juice the following experiment was performed.

Thirty-six lemons were thoroughly scrubbed and washed in a hot detergent solution and rinsed with water. The lemons were then allowed to soak in hot water for about $30 \mathrm{~min}$.

The epicarp (flavedo) was carefully strippeu from each fruit by means of a stainless steel knife, and the fruit rinsed in cool water. The mesocarp (albedo) and segment membranes of the fruit were then removed and the lemons quartered. Finally the central vascular bundle and all the seeds were removed.

The sectioned juice sacs were homogenized in a blendor for $15 \mathrm{~min}$., and the homogenate filtered to remove any remaining membrane material. $250 \mathrm{gm}$. of this material were extracted with three $100-\mathrm{ml}$. portions of low-boiling petroleum ether, the extracts combined, dried over anhydrous sodium sulphate, filtered and the solvent removed under reduced pressure on a steam-bath. A pale yellow oil resulted. A small amount of the oil (about $0.01 \mathrm{ml}$.) was applied to a silicic acid chromatostrip ${ }^{6}$ and irrigated with a solution of 25 per cent ethyl acetate in $n$-hexane. Examination of the strip under ultra-violet light (2540 A.) revealed eight distinct spots or zones.

Several downward chromatostrips ${ }^{5}$ were run, the individual zones scraped from the strip, each eluted with 95 per cent ethanol and the eluates filtered. The ultra-violet spectrum of each eluate was then taken.

Comparison of $R_{F}$ values with known coumarin compounds and ultra-violet absorption spectra tentatively identified five of these compounds as 5geranoxypsoralen (bergamottin), 8-geranoxypsoralen, 5,7-dimethoxycoumarin (limettin), oxypeucedanin hydrate and byakangelicin (Table 1).

To date, insufficient amounts of these compounds have been isolated from the juice for more precise analytical examination; but this work is in progress. Richard A. Bernhard

Department of Food Technology,

University of California,

Davis, California. July 24.

1 Burgess, H. E., Chem. Z., 25, 602 (1901).

'Schmidt, E., Apoth. Z., 16, 619 (1901).

${ }^{3}$ Rodighiero, G., Caporale, G., and Ragazzi, E., Atti Ist. Veneto sci. Lettere e Arti, Classe Sci., Mat. e. Nat., 3, $125(1952-1953)$; Chem. Abstracts, 48, 14116h (1954).

- Stanley, W, L., and Vannier, S. H., J. Amer. Chem. Soc., 79, 3488 (1957).

- Miller, J. M., and Kirchner, J. G., Anal. Chem., 26, 2002 (1954).

- Stanley, W. L., and Vannier, S. H., J. Assoc. Official Agric. Chem., 582 (1957).

'Rice, R. G., Keller, G. J., and Beavens, E. A., Food Tech., 6, 35 (1952). 